> Les jonctions serrées scellent les cellules épithéliales et endothéliales entre elles. Elles ont été longtemps considérées comme de simples barrières séparant les deux compartiments membranaires apical et basolatéral, dont la composition en protéines et lipides est différente. De récentes études montrent que diverses protéines impliquées dans la transduction de signaux et le trafic membranaire sont associées aux jonctions serrées. Ces dernières servent de lieu d'intégration et de transmission de signaux nécessaires à la régulation de leur assemblage et à la polarisation cellulaire, mais aussi à la modulation de l'expression des gènes requise pour la prolifération et la différenciation cellulaires. <

\section{Les jonctions serrées \\ Plate-forme de régulation de la prolifération et de la polarité cellulaires}

Ahmed Zahraoui

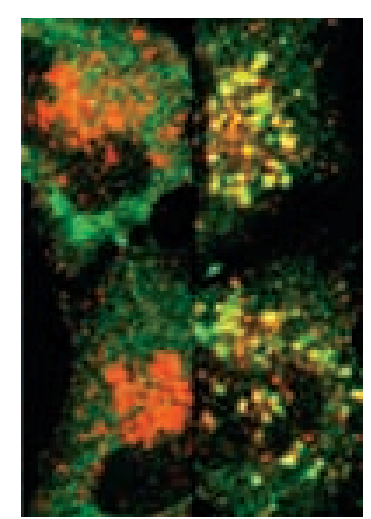

d'un réseau continu de fibres ramifiées et anastomosées qui entoure l'apex de chaque cellule. Ces fibres sont constituées de polymères de protéines membranaires, les claudines et l'occludine. est différente. Les jonctions serrées séparent la membrane apicale du domaine basolatéral. Elles forment une barrière sélective régulant le passage des ions et molécules à travers l'espace paracellulaire. En empêchant les protéines et lipides de la membrane apicale de diffuser dans la membrane basolatérale, et inversement, elles contribuent au maintien de la polarité cellulaire. Des études récentes ont mis en évidence la complexité des jonctions serrées, qui forment un échafaudage macromoléculaire composé de protéines membranaires et cytoplasmiques essentielles au maintien de l'adhérence cellule-cellule et au contrôle de la prolifération, de la différenciation et de la polarité cellulaires.

\section{Structure et organisation moléculaire des jonctions serrées}

Les jonctions serrées se présentent en microscopie électronique comme une série de points de fusion apparente des feuillets externes des membranes plasmiques de deux cellules adjacentes. Ces zones de contact membranaires apparaissent, par cryofracture, sous forme
Laboratoire de morphogenèse et signalisation cellulaires, CNRS UMR 144, Institut Curie, 26, rue d'Ulm, 75248 Paris, Cedex 05, France.

zahraoui@curie.fr

\section{Protéines membranaires}

Quatre types de protéines membranaires, claudines, occludine, JAM (junctional adhesion molecule) et Crumb ont été identifiées. Les claudines, qui composent une famille d'au moins 24 membres, sont responsables de la formation des jonctions serrées. Au moins deux claudines différentes sont exprimées dans chaque cellule, la claudine 5 l'étant principalement dans les cellules endothéliales et la claudine 11 dans les cellules de Sertoli et les oligodendrocytes.

Les claudines et l'occludine possèdent quatre domaines transmembranaires. Elles interagissent via leurs domaines extracellulaires avec celles de la cellule voisine pour établir le contact cellule-cellule. Les claudines sont capables de se polymériser et de former des pores qui permettent une diffusion sélective des ions et molécules à travers l'espace paracellulaire. La perméabilité sélective de ces canaux dépend de la concentration et du type de claudine exprimé par la cellule épithéliale ou endothéliale: par exemple, la claudine 16 
forme des canaux pour le $\mathrm{Mg}^{2+}$ dans la branche large ascendante de l'anse de Henle, tandis que la claudine 4 forme des canaux pour les ions $\mathrm{Na}^{+}$. Bien que de nombreuses études menées sur les cellules épithéliales en culture aient indiqué l'importance de l'occludine dans la fonction de barrière des jonctions serrées, son rôle n'est pas encore clairement établi $[1,2]$.

Les protéines JAM, qui composent une famille de trois protéines, possèdent un seul domaine transmembranaire; elles régulent la migration des monocytes à travers les cellules endothéliales et seraient impliquées dans la formation des jonctions intercellulaires. Contrairement aux claudines et à l'occludine, JAM est exprimée dans les plaquettes et les leucocytes [3]. Crumb (Crb), protéine présentant un domaine transmembranaire unique, est exprimée dans les neurones, les cellules épithéliales et les cellules photoréceptrices. Trois Crb ont été identifiées chez l’homme, dont la protéine Crb3 associée aux jonctions serrées et à la membrane apicale [4].

\section{Protéines de la plaque cytoplasmique}

Les protéines membranaires (claudines, occludine et JAM) constituent le noyau d'un échafaudage moléculaire relié à une multitude de protéines cytoplasmiques. Celles-ci peuvent être réparties en deux groupes: le premier est composé de protéines à domaines PDZ (PSD95 [postsynaptic density 95], DIg [discs large] et 20-1) telles que Z0-1 à 3 (zonula occludens), MUPP 1 (multi-PDZ domain protein 1), Par-3/ASIP (partitioning defective/atypical PKC isotype-specific interacting protein), Par-6, Pals (protein associated with Lin-7), MAGI-1 à 3 (membrane associated guanylate kinase with inverted domain structure) (Figure 1). Les domaines PDZ sont capables de reconnaître des motifs de trois acides aminés (Ser/Thr-X-Val/Ile) situés à l'extrémité carboxyterminale des protéines membranaires $[5,6]$. Les protéines à domaines PDZ sont des adaptateurs moléculaires permettant la liaison de diverses protéines membranaires au cytosquelette d'actine ou à des protéines régulatrices. Le second groupe de protéines cytoplasmiques impliquées dans la formation des jonctions serrées contient différents types de régulateurs: Rab13, Cdc42 (cell-division control protein 42), cinguline, symplekine, PKCa (PKC atypique), ZONAB (Z0-1 associated nucleic acid protein), huASHl (absent, small or homeotic discs 1) ou SAF-B (scaffold attachment factor-B).

\section{Formation des jonctions serrées}

Les phénomènes impliqués dans l'assemblage des jonctions serrées semblent être très similaires dans les différents

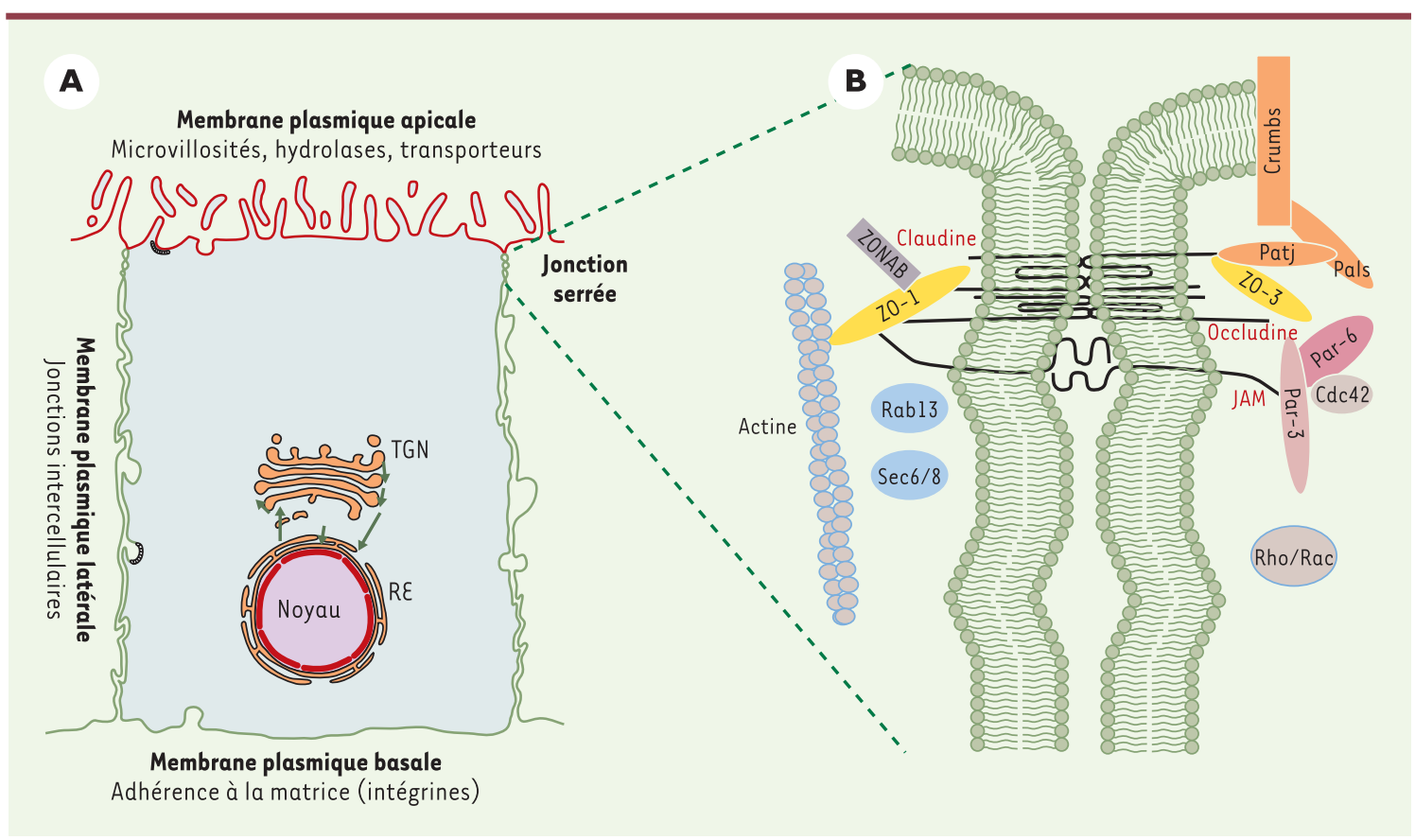

Figure 1. Organisation des complexes protéiques au sein des jonctions serrées. A. La position des jonctions serrées délimite la membrane apicale de la membrane basolatérale d'une cellule. RE: réticulum endoplasmique; TGN: trans-Golgi network. B. Composants et organisation des complexes protéiques constituant les jonctions serrées. Z0- 1 et 3 : zonula occludens 1 et 3 ; ZONAB: Z0-1 associated nucleic acid protein; rab13: petite protéine G; Sec6/8: complexe de sécrétion; JAM: junctional adhesion molecule; Pals: protein associated with Lin-7; Patj: Pals associated tight junction; Par-3 et 6: partitioning defective 3 et 6 ; Cdc 42 : cell-division control protein 42 ; rab, rho et rac: petites protéines $G$. 
modèles cellulaires. Le modèle le plus étudié est celui de la cellule épithéliale en culture MDCK (Madin-Darby canine kidney).

Après l'établissement de contacts cellule-cellule par le biais de la $\varepsilon$-cadhérine, on observe la mise en place d'un complexe jonctionnel contenant les protéines des jonctions adhérentes et serrées. L'activation de protéines régulatrices comme les petites protéines $G$ Cdc42 (cell-division control protein) ou Rabl3 induit la stabilisation des contacts intercellulaires et l'activation de la protéine kinase $C$ atypique ( $P K C a$ ). Celle-ci joue un rôle déterminant dans la régulation de l'assemblage des jonctions serrées. Très conservée au cours de l'évolution, la PKCa forme un complexe avec

Par-3 et Par-6 (partitioning defective). Les gènes Par, d'abord identifiés chez le nématode $C$. elegans, régulent la localisation asymétrique de plusieurs protéines, l'orientation du fuseau mitotique et la division asymétrique. Par-6 contient un domaine CRIB ( $C d c 42 /$ rac interactive binding) capable de se lier à la forme active de $\mathrm{Cdc42}$ [7], qui régulerait l'activation du complexe Par6/Par3/PKCa nécessaire pour promouvoir la maturation des jonctions serrées fonctionnelles.

Chez les mammifères, la protéine JAM sert de point d'ancrage à Par-3, permettant ainsi le recrutement du complexe Par-6/Par-3/PKCa au niveau des jonctions serrées [8]. Ce dernier stimule la formation de deux jonctions, adhérente et serrée, séparées et matures. La PKCa est régulée négativement par la protéine phosphatase 2A (PP2A), qui la déphosphoryle. L'expression de la sous-unité catalytique de PP2A dans les cellules MDCK provoque la déphosphorylation des protéines des jonctions serrées $20-1$, occludine et claudine 1 , et inhibe l'assemblage des jonctions serrées. Inversement, l'inhibition de PP2A par l'acide okadaïque stimule la phosphorylation et le recrutement des protéines $20-1$, occludine et claudine 1 aux jonctions serrées [9]. Ces résultats mettent en évidence le rôle des épisodes de phosphorylation/déphosphorylation, via la PKCa et la PP2A, dans l'assemblage et le désassemblage des jonctions serrées.

Enfin, trois familles de petites protéines $G$ sont impliquées dans la régulation des jonctions serrées: Ras, Rho et Rab. La production des formes actives de Rac et Rho altère les fonctions des jonctions serrées. Elle provoque, dans les cellules MDCK, la désorganisation et une redistribution anarchique de l'occludine et de Z01 [10]. Étant donné le rôle crucial de Rho et de Rac dans la dynamique du cytosquelette d'actine et l'importance de ce dernier dans l'établissement et le maintien de l'intégrité des jonctions serrées, il est probable que ces deux protéines $G$ altèrent localement le réseau d'actine-myosine requis pour l'immobilisation de complexes macromoléculaires au niveau des jonctions serrées.

\section{Rôle des jonctions serrées dans la prolifération et la différenciation cellulaires}

L'implication des protéines des jonctions serrées dans la prolifération et la différenciation cellulaires a été d'abord montrée chez la drosophile. Les mutations inactivant dlgA (discs large), une protéine de la même famille que $20-1$ associée aux jonctions septées (Figure 2), provoquent la perte de la polarité cellulaire et la croissance anarchique de cellules épithéliales dans les disques imaginaux de la larve [11]. Ces résultats suggèrent que dlgA contrôle négativement la prolifération des cellules épithéliales.

L'expression de formes tronquées de $20-1$ induit la dédifférenciation et la transformation de la cellule

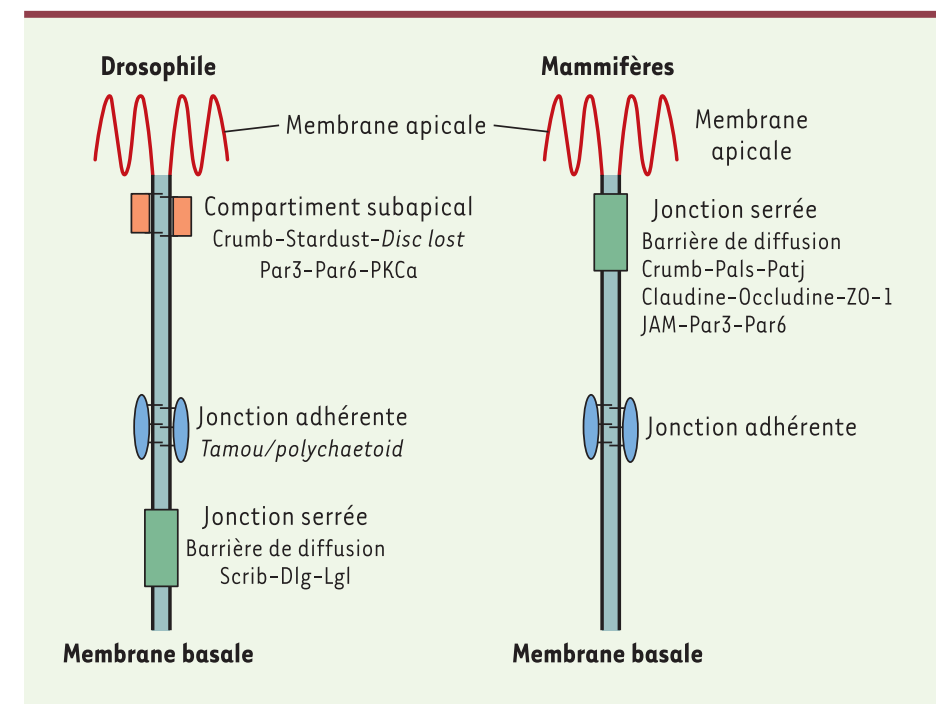

Figure 2. Comparaison des jonctions intercellulaires des cellules épithéliales chez la drosophile et chez les mammifères. Seules les jonctions adhérentes sont structurellement et fonctionnellement conservées entre la drosophile et les mammifères. Bien qu'elles constituent une barrière de diffusion, les jonctions serrées et septées diffèrent par leur position sur la membrane latérale et par leur composition en protéines. En effet, les homologues des jonctions serrées chez la drosophile sont associés avec les jonctions adhérentes tamou/polychaetoid (homologue de Z0-1), ou avec le compartiment subapical. Crumb est quant à elle localisée le long de la membrane apicale, aussi bien chez la drosophile que chez les mammifères. Les jonctions serrées peuvent être considérées comme l'équivalent fonctionnel des jonctions septées et du compartiment subapical. Chez la drosophile, c'est le compartiment subapical qui sépare la membrane apicale de la membrane basolatérale. 20-1: zonula occludens 1; JAM: junctional adhesion molecule; Pals : protein associated with Lin-7; Patj: Pals associated tight junction; Par-3 et -6: partitioning defective 3 et 6 ; PKCa: PKC atypique; Srib-DIg-DIt: scribble-lethal giant larvae-discs lost. 
épithéliale en cellule mésenchymateuse [12]. La découverte des protéines des jonctions serrées simplekine, 20-1 et 20-2 dans le noyau des cellules prolifératives, et surtout des facteurs de transcription huASHl, ZONAB et SAF-B associés aux jonctions serrées, a permis d'impliquer ces structures dans la régulation de l'expression des gènes. La simplekine interviendrait dans la maturation de I'ARN prémessager, alors que le facteur ASHI de drosophile a une activité méthyltransférase sur les histones. Dans les cellules MDCK, ZO-1 et 20-2 interagissent respectivement avec les facteurs de transcription ZONAB et SAF-B. L'interaction de $20-1 / Z O N A B$ régule l'expression du gène $c$ erb2 codant pour le corécepteur du facteur de croissance épithélial $\varepsilon G F$, qui a un rôle important dans la différenciation des cellules épithéliales [13-17]. II semble que $20-1$ ne participe pas directement à la régulation de l'expression de c-erb2, mais serve à séquestrer ZONAB dans les jonctions serrées des cellules épithéliales polarisées. Dans le noyau, ZONAB interagit avec la CDK4 (cyclin-dependent kinase 4) requise pour la transition de la phase $G l$ à la phase $S$

\begin{tabular}{ll}
\hline $\begin{array}{l}\text { Protéine } \\
\text { Protéines membranaires }\end{array}$ & \multicolumn{1}{l}{ Fonction } \\
$\begin{array}{l}\text { Claudines } \\
\text { Occludine }\end{array}$ & Perméabilité paracellulaire \\
JAM & Maturation des jonctions serrées \\
Crumb & Polarité \\
Protéines d'échafaudage & \\
Z0-1, Z0-2, Z0-3 & \\
MUPP1 & Assemblage des jonctions serrées, \\
MAGI-1, -2 et -3 & polarité et différenciation \\
Pals1 & \\
$\begin{array}{l}\text { Patj } \\
\text { ASIP/Par-3 }\end{array}$ & \\
Par-6 & \\
Protéines régulatrices & \\
PKCa, PP2A & Assemblage des jonctions serrées \\
Cdc42 & Trafic membranaire \\
Rabl3 & Arrimage des vésicules de transport \\
Sec6, Sec8 & \\
\hline
\end{tabular}

Tableau I. Principales protéines membranaires, d'échafaudage et régulatrices des jonctions serrées chez les mammifères. JAM: junctional adhesion molecule; Z0-1 à 3: zonula occludens 1 à 3 ; MUPP1: multi-PDZ domain protein 1; MAGI-1 à 3: membrane associated guanylate kinase with inverted domain structure 1 à 3; Pals: protein associated with Lin-7; Patj: Pals associated tight junction; ASIP/Par-3: atypical PKC isotype-specific interacting protein/partitioning defective; PKCa: PKC atypique; PP2A: protéine phosphatase 2A; Cdc42: cell-division control protein 42. du cycle cellulaire [18]. Par conséquent, en séquestrant ZONAB dans les jonctions serrées, $20-1$ régule négativement l'expression de c-erb2 et la prolifération cellulaire.

Les jonctions serrées sont également les cibles de certains effecteurs de Ras, en particulier des MAP-kinases (mitogen activated protein kinases). L'expression des formes actives de Raf-1, MAPK ou ERK kinase (MEKI) est suffisante pour dédifférencier les cellules épithéliales. Le traitement des cellules MDCK transformées par Ras avec un inhibiteur de MEKl permet la réversion et la ré-acquisition du phénotype épithélial. De même, l'accumulation de l'occludine dans les jonctions serrées inhibe les voies de signalisation Raf-l et $\beta$-caténine impliquées dans la prolifération et rétablit l'adhésion intercellulaire [19, 20]. L'introduction dans les cellules MDCK d'un peptide correspondant au deuxième domaine extracellulaire de l'occludine entraîne la dissociation de l'occludine des jonctions serrées et l'accumulation de la $\beta$-caténine dans le cytoplasme. Celle-ci s'associe au facteur de transcription TCF/LEF ( $T$-cell factor- lymphoid enhancer-binding factor) et stimule l'expression du gène $c-m y c$ impliqué dans la prolifération cellulaire. La $\beta$-caténine régule également la transcription du gène claudine 1 .

Ces observations ont permis de proposer que les jonctions serrées soient des modulateurs de la densité cellulaire. Dans les cellules épithéliales en croissance, le niveau d'expression des protéines des jonctions serrées est faible, ce qui a un effet prolifératif. À l'inverse, l'augmentation de la densité cellulaire stimule l'expression et l'accumulation des protéines des jonctions serrées, avec pour corollaire le recrutement et la séquestration de facteurs de transcription et l'inhibition de la prolifération.

\section{Rôle des jonctions serrées dans l'établissement et le maintien de la polarité}

Les jonctions serrées sont requises pour maintenir la polarité des cellules épithéliales et endothéliales. Des travaux déjà anciens ont suggéré que les jonctions intercellulaires constituent un domaine préférentiel d'arrimage de vésicules de transport [21]. De fait, les petites protéines $\mathrm{G}$ Rab3b, Rab8 et Rabl3, qui régulent les différentes étapes du trafic membranaire, sont localisées au niveau des jonctions serrées. Elles coordonneraient l'assemblage de complexes protéiques requis pour l'adressage et l'arrimage des vésicules de transport avec les membranes cibles [5]. Nous avons montré que l'expression de la forme active (liant le 
GTP) de Rabl3 altère la structure et la perméabilité paracellulaire des jonctions serrées, ainsi que la polarité des cellules épithéliales. Rabl3 régule la localisation de la claudine 1 nécessaire pour former des jonctions serrées fonctionnelles [22]. Comme Rabl3, le complexe $\operatorname{Sec} 6 / \mathrm{Sec} 8$ (secretion gene products), impliqué dans l'arrimage des vésicules de transport à la membrane basolatérale, est associé aux jonctions serrées des cellules MDCK. Le recrutement du complexe Sec6/Sec8 et des protéines Rab, avec leurs effecteurs, à proximité des jonctions serrées permettrait l'organisation d'un microdomaine membranaire où des vésicules cargos chargées de protéines basolatérales ou apicales sont préférentiellement livrées. Cependant, la question cruciale des mécanismes permettant la restriction et le maintien des protéines apicales et basolatérales dans leurs domaines respectifs demeure.

Des études récentes montrent l'existence de complexes protéiques très conservés au cours de l'évolution associés aux jonctions intercellulaires (Tableau II). Chez la drosophile, les complexes protéiques Crb, Baz (Bazooka) et Scrib (Scribble) coopèrent pour établir et maintenir la polarité des cellules épithéliales. Le complexe Scrib, associé aux jonctions adhérentes, empêche l'extension du domaine apical et inhibe l'action des complexes $\mathrm{Crb}$ et Baz. Par conséquent, les complexes Crb-Sdt-Dlt (Stardust-Discs lost) et Baz-Par6-PKCa, d'une part, et Scrib-Lgl-DIg (lethal giant larvae), d'autre part, agissent de façon compétitive pour délimiter les membranes apicale et basolatérale et positionner correctement les jonctions intercellulaires [4, $23]$. Chez les mammifères, ces complexes contribueraient à la mise en place et au maintien de la polarité cellulaire [24, 25].

\section{Jonctions serrées et maladies humaines}

Plusieurs carcinomes sont associés à une perte de polarité cellulaire et à la dissociation des jonctions serrées. Par exemple, la claudine 1 est fortement exprimée dans plusieurs cancers colorectaux [26], et la claudine 4 dans les cancers de l'ovaire. De plus, les oncogènes Ras et Raf-l entraînent la délocalisation de l'occludine et la dissociation des jonctions serrées. Ces arguments révèlent que la dissociation des jonctions serrées est une étape clé dans la progression tumorale.

Les mutations affectant le gène claudine 14 provoquent une surdité autosomique récessive. La claudine 14 est exprimée dans l'épithélium sensoriel de l'organe de Corti. La cochlée de l'oreille interne possède deux com- partiments de composition ionique différente, la périlymphe et l'endolymphe, et la claudine 14 est essentielle pour maintenir le gradient électrochimique entre ces deux compartiments.

L'occludine, les claudines ainsi que les protéines JAM et Z0-1 sont souvent les cibles de toxines bactériennes. Par exemple, lors de l'infection par Helicobacter pylori des cellules épithéliales gastriques, la protéine bactérienne CagA (cytotoxic-associated gene A) s'associe avec $20-1$ et JAM et altère l'organisation et la fonction des jonctions serrées [27, 28].

\section{Conclusions et perspectives}

Les données présentées dans cet article montrent que les jonctions serrées servent de lieu d'intégration et de transduction de signaux nécessaires pour réguler l'adhérence, la prolifération, la différenciation et la polarité cellulaires. Les récentes études ont amélioré considérablement notre connaissance sur la composition et l'activité des jonctions serrées. Cependant, nous sommes encore loin de comprendre comment les différents complexes des jonctions serrées interagissent les uns avec les autres pour promouvoir l'établissement et le maintien des jonctions serrées, ainsi que la polarité. Les approches tendant à déterminer les fonctions des gènes par interférence $A R N$, de même que la construction de cartes d'interactions protéine-protéine, devraient permettre d'identifier de nouveaux candidats et contribuer à disséquer les séquences d'événements contrôlant la polarité cellulaire. Ces études devraient non seulement faciliter la compréhension de la polarité, mais aussi celle des causes de certaines maladies malignes (carcinomes associés à la perte de la polarité) ou dégénératives, touchant la rétine (retinitis pigmentosa associée à des mutations de Crumb). $\diamond$

\begin{tabular}{ll}
\hline Drosophile & Mammifères \\
Crb (Crumb-Stardust-DIt) & Crb (Crumb-Pals-Patj) \\
Baz (Baz-Par-6-PKCa) & Par3 (Par-3/ASIP-Par-6-PKCa) \\
Scrib (Scrib-DIg-LgI) & Erb (Erbin-DIg-MLgI)
\end{tabular}

Tableau II. Complexes protéiques des jonctions serrées de drosophile, et leurs homologues chez les mammifères. Pals: protein associated with Lin-7; Patj: Pals associated tight junction; ASIP/Par-3: atypical PKC isotype-specific interacting protein/partitioning defective; PKCa: PKC atypique. 


\section{SUMMARY}

Tight junctions, a platform regulating cell

proliferation and polarity

Tight junctions (TJ) are specialized plasma membrane microdomains that encircle the apical pole of each epithelial and endothelial cell, separating apical from basolateral side. They form an intercellular diffusion barrier (or gate) regulating the passage of ions, water, and various macromolecules through the paracellular spaces, and a fence restricting the apical/basolateral diffusion of membrane proteins and lipids. This latter function is deeply involved in cancer cell biology, in terms of loss of cell polarity. Several TJ proteins are involved in organizing signal transduction at TJ. For example, the interaction of TJ protein 20-1 (zonula occludens 1) with the transcription factor ZONAB (ZO-1 associated nucleic acid protein) is important in the regulation of expression of the proto-oncogene $\varepsilon r b-2$ as well as epithelial proliferation/differentiation. Recent studies indicate that different proteins involved in membrane trafficking are associated with tight junctions. Among these proteins are the small G-proteins of the Rab family that regulate specific membrane transport events in both endocytic and exocytic pathways. Rab proteins may coordinate the recruitment of protein complexes necessary for the establishment-maintenance of cell polarity. Based on the studies reviewed here, tight junctions emerge as a platform used to coordinate and regulate cell polarity, and proliferation/differentiation. $\diamond$

\section{RéFÉRENCES}

1. Tsukita S, Furuse M. Occludin and claudins in tight-junction strands: leading or supporting players? Trends Cell Biol 1999; 9: 268-73.

2. Tsukita S, Furuse M. Claudin-based barrier in simple and stratified cellular sheets. Curr Opin Cell Biol 2002; 14: 531-6.

3. Martin-Padura I, Lostaglio S, Schneemann $M$, et al. Junctional adhesion molecule, a novel member of the immunoglobulin superfamily that distributes at intercellular junctions and modulates monocyte transmigration. J Cell Biol 1998; 142:117-27.

4. Knust $\varepsilon$, Bossinger 0 . Composition and formation of intercellular junctions in epithelial cells. Science 2002; 298: 1955-59.

5. Zahraoui A, Louvard D, Galli T. Tight junction, a platform for trafficking and signaling protein complexes. J Cell Biol 2000; 151: F31-6.

6. Matter K, Balda MS. Signalling to and from tight junctions. Nat Rev Mol Cell Biol 2003; 4 : 225-36.

7. Joberty G, Petersen C, Gao L, Macara IG. The cell-polarity protein Par6 links Par3 and atypical protein kinase C to Cdc42. Nat Cell Biol 2000; 2: $531-9$.

8. Itoh M, Sasaki H, Furuse M, et al. Junctional adhesion molecule (JAM) binds to PAR-3: a possible mechanism for the recruitment of PAR-3 to tight junctions. J Cell Biol 2001; 154 : 491-8.

9. Nunbhakdi-Craig V, Machleidt T, Ogris $\varepsilon$, et al. Protein phosphatase $2 \mathrm{~A}$ associates with and regulates atypical PKC and the epithelial tight junction complex. J Cell Biol 2002; 26: 26.
10. Jou TS, Schneeberger દદ, Nelson WJ. Structural and functional regulation of tight junctions by RhoA and Racl small GTPases. J Cell Biol 1998; 142: 101-15.

11. Willott $\varepsilon$, Balda MS, Fanning AS, et al. The tight junction protein $20-1$ is homologous to the Drosophila discs-large tumor suppressor protein of septate junctions. Proc Natl Acad Sci USA 1993; 90: 7834-8.

12. Ryeom SW, Paul D, Goodenough DA. Truncation mutants of the tight junction protein Z0-1 disrupt corneal epithelial cell morphology. Mol Biol Cell 2000; 11: 1687-96.

13. Nakamura T, Blechman J, Tada S, et al. HuASHl protein, a putative transcription factor encoded by a human homologue of the Drosophila ashl gene, localizes to both nuclei and cell-cell tight junctions. Proc Natl Acad Sci USA 2000; 97 : 7284-9.

14. Traweger A, Fuchs R, Krizbai IA, et al. The tight junction protein ZO-2 localizes to the nucleus and interacts with the hnRNP protein SAF-B. J Biol Chem 2002; $27: 27$.

15. Balda MS, Matter K. The tight junction protein $20-1$ and an interacting transcription factor regulate ErbB-2 expression. EMBO J 2000; 19 : 2024-33.

16. Gottardi CJ, Arpin M, Fanning AS, Louvard D. The junction-associated protein, zonula occludens- 1 , localizes to the nucleus before the maturation and during the remodeling of cell-cell contacts. Proc Natl Acad Sci USA 1996; 93: 10779-84.

17. Hofmann I, Schnolzer M, Kaufmann I, Franke WW. Symplekin, a constitutive protein of karyo- and cytoplasmic particles involved in mRNA biogenesis in Xenopus laevis oocytes. Mol Biol Cell 2002; 13: 1665-76.

18. Balda MS, Garrett MD, Matter K. The Z0-1-associated Y-box factor ZONAB regulates epithelial cell proliferation and cell density. J Cell Biol 2003; 160: 423-32.

19. Li D, Mrsny RJ. Oncogenic Raf-1 disrupts epithelial tight junctions via downregulation of occludin. J Cell Biol 2000; 148: 791-800.

20. Montesano R, Soriano JV, Hosseini G, et al. Constitutively active mitogen-activated protein kinase kinase MEKI disrupts morphogenesis and induces an invasive phenotype in Madin-Darby canine kidney epithelial cells. Cell Growth Differ 1999; 10 : 317-32.

21. Louvard D. Apical membrane aminopeptidase appears at site of cell-cell contact in cultured kidney epithelial cells. Proc Natl Acad Sci USA 1980; $77: 4132-6$.

22. Marzesco AM, Dunia I, Pandjaitan R, et al. The small GTPase Rabl3 regulates assembly of functional tight junctions in epithelial cells. Mol Biol Cell 2002; 13: 1819-31.

23. Bilder D, Schober M, Perrimon N. Integrated activity of PDZ protein complexes regulates epithelial polarity. Natl Cell Biol 2003; $5: 53-8$.

24. Lemmers C, Medina $\varepsilon$, Delgrossi MH, et al. HINADI/PATJ, a homolog of discs lost, interacts with crumbs and localizes to tight junctions in human epithelial cells. J Biol Chem 2002; 277: 25408-15.

25. Roh MH, Makarova 0, Liu CJ, et al. The Maguk protein, Palsl, functions as an adapter, linking mammalian homologues of Crumbs and Discs lost. J Cell Biol 2002; 157: 161-72.

26. Miwa N, Furuse M, Tsukita S, et al. Involvement of claudin-l in the betacatenin/Tcf signaling pathway and its frequent upregulation in human colorectal cancers. Oncol Res 2001; 12: 469-76.

27. Amieva MR, Vogelmann R, Covacci A, et al. Disruption of the epithelial apical-junctional complex by Helicobacter pylori CagA. Science 2003; $300: 1430-4$.

28. Sawada N, Murata M, Kikuchi K, et al. Tight junctions and human diseases. Med Electron Microsc 2003; 36: 147-56.
TIRÉS À PART

A. Zahraoui 